\title{
Clarifying the onset of brief psychotic disorder at childbirth
}

\author{
Verinder Sharma $^{1,2} \cdot$ Christine Baczynski $^{2}$ \\ Received: 28 January 2019 / Accepted: 29 March 2019 / Published online: 6 April 2019 \\ (C) Springer-Verlag GmbH Austria, part of Springer Nature 2019
}

\section{Clarifying the onset of brief psychotic disorder at childbirth}

Brief psychotic disorder is characterized by a sudden onset of symptoms including delusions, hallucinations, disorganized speech, or catatonia. The disorder is known for its brevity with symptoms lasting between 1 and 30 days (American Psychiatric Association 2013). Duration of symptoms is the feature that distinguishes it from schizophreniform disorder (1 to 6 months) and schizophrenia (at least 6 months). A large number of patients with brief psychotic disorder experience a change in diagnosis to another psychotic disorder or a mood disorder (Schwartz et al. 2000) over time. Individuals with the disorder usually make a good recovery with full return to premorbid levels of functioning; however, the usual lack of a prodrome poses a challenge for early detection and prompt management of the disorder.

The DSM-5 allows the use of two specifiers to signify the onset of psychiatric disorders during and/or after pregnancy. The peripartum onset specifier is applied to denote mood episodes in women with major depressive disorder, bipolar I disorder, or bipolar II disorder if there is onset of a mood episode during pregnancy or within the first 4 weeks postpartum (American Psychiatric Association 2013). The postpartum onset specifier is recommended to signify the onset of brief psychotic disorder; however, the DSM-5 text defines this specifier as onset of psychosis during pregnancy or within 4 weeks postpartum. This represents an inconsistency in terminology.

The purpose of this letter is to alert clinicians of the discrepancy between the specifier for brief psychotic disorder and its definition in the DSM-5. In order to resolve the discrepancy, "postpartum specifier" should be replaced with "peripartum specifier" to allow the characterization of pregnancy-onset psychotic episodes. Alternatively, the definition of postpartum onset should be changed to reflect a period of time following childbirth and not during pregnancy. It is hoped that these inconsistencies will be rectified in future publication of the DSM-5, and in future DSM iterations.

\section{Compliance with ethical standards}

Conflict of interest Dr. Sharma has received grant support from, participated on scientific advisory boards for, or served on speakers' bureaus of Abilify Maintena, Assurex, Genome Canada, Lundbeck, Neurocrine Biosciences, Otsuka, Sage Therapeutics, Stanley Medical Research Institute, and Sunovion Pharmaceuticals. Ms. Baczynski reports no conflict of interest.

\section{References}

American Psychiatric Association (2013) Diagnostic and statistical manual of mental disorders, 5th edn. Author, Washington

Schwartz JE, Fennig S, Tanenberg-Karant M, Carlson G, Craig T, Galambos N, Lavelle J, Bromet EJ (2000) Congruence of diagnoses 2 years after a first-admission diagnosis of psychosis. Arch Gen Psychiatry 57(6):593-600

Publisher's note Springer Nature remains neutral with regard to jurisdictional claims in published maps and institutional affiliations.
Verinder Sharma

vsharma@uwo.ca

1 Western University, London, Ontario, Canada

2 Parkwood Institute, Mental Health Care Building, London, Ontario, Canada 